DOI 10.4467/2543733XSSB.17.012.7258

LIDWINE WARCHOL

Université Paris-Pantheon \& CNRS

\title{
LA POLOGNE EN FRANCE: LES DIFFÉRENCES DE PERCEPTION ENTRE L'OPINION PUBLIQUE ET LES INTELLECTUELS FRANÇAIS ${ }^{1}$
}

Les mots-clés: Pologne, France, intellectuels, exilés politiques, relations reciproques

Le sujet proposé cherche à étudier comment ont été perçus, par différentes catégories sociales et à différents moments historiques, la Pologne et les Polonais en France, en fonction d'un contexte français. Cette approche s'inscrit dans l'étude des transferts culturels qui, comme l'a montré Michael Werner dans ses articles précurseurs ${ }^{2}$, supposent un point de départ et un même point d'arrivée. Ici le point de départ est celui de la France et il s'agit de voir, à partir de ce que vivent les Français, ce qu'a modifié pour eux la rencontre avec la Pologne. L'histoire croisée étudie en effet la manière dont un individu s'imprègne ou non par la relation. Et pour enclencher le contact, il faut un contexte personnel, une accroche idéologique, politique, qui donne une impulsion vers l'autre.

Tous les pays, les peuples véhiculent sur les autres peuples des représentations, des stéréotypes qui se construisent avec le temps et des contacts. Ces images qui se dessinent sur l'autre, l'étranger, sont souvent dépendantes en réalité du pays d'où l'on parle. Transférer des événements extérieurs suppose de les réutiliser. En ce qui concerne la perception de la Pologne en France, celle-ci a varié au cours du temps en fonction d'enjeux de différentes natures. Assez unanimement, il est admis en France que les premières relations entre la France et la Pologne datent de la fin du XVI ${ }^{\mathrm{e}}$ siècle, lorsque les deux pays sont gouvernés par le même roi, Henri de Valois. Mais ce même événement peut donner lieu à des interprétations différentes en fonction des époques et en fonction de ce que l'on veut montrer du rapport de la France à la Pologne. Soit il s'agit de mettre en avant un lien très fort, et dans ce cas ce premier contact est valorisé comme un moment initiatique qui

\footnotetext{
${ }^{1}$ Article publié dans le cadre du projet soutenu par le Comité National des Sciences: NCN no 2013/08/M/ HS3/00395»

${ }^{2}$ M. Werner, B. Zi mmerman n, «Penser l'histoire croisée: entre empire et réflexivité», Annales ESC (ou Annales. Economies, Sociétés, Civilisations), janvier-février 2003, n 1, p. 7-36 ; (dir), De la comparaison à l'histoire croisée, Le genre humain, EHESS, 2004.
} 
marque l'essor de la présence française à la cour polonaise. Soit il s'agit d'exprimer une désillusion au sujet de relations entre la France et la Pologne bien moins solides au cours de l'histoire que l'on ne croit et dans ce cas, c'est la fuite du roi au bout de huit mois que l'on soulignera.

Cette étude ne propose pas d'analyser de manière exhaustive les relations franco-polonaises, ce qui par ailleurs a déjà pu être fait, ne serait-ce que dans des ouvrages collectifs de référence en France comme celui de Bernard Michel et Józef Łaptos sur les relations franco-polonaises ou sur les contacts franco-polonais depuis le Moyen-Âge au niveau religieux ${ }^{3}$. L'idée est plutôt de se concentrer sur la perception de la Pologne au niveau des deux entités que sont les intellectuels et l'opinion publique, afin de montrer en quoi l'origine ou la fonction sociale peut différencier la perception de l'étranger. Le terme d'intellectuel, à caractère très polémique dans de nombreux pays, sera utilisé dans ce sujet à partir de deux aspects interdépendants, aspects proposés dans les nombreux travaux en France sur le sujet ${ }^{4}$. D'abord, l'intellectuel se définit comme un individu ayant acquis une reconnaissance sociale et une position parmi les élites culturelles d'un pays du fait de ses travaux, de ses publications. D'autre part, ce chercheur, cet écrivain, cet universitaire peut prétendre au statut d'intellectuel à partir du moment où il se sert du capital de popularité acquis par ses travaux auprès de l'opinion publique pour intervenir et s'engager pour des causes universelles telles que la justice, la liberté ou les droits de l'Homme. Cet engagement se manifeste pour la première fois au moment de l'Affaire Dreyfus en France, à la fin du $\mathrm{XIX}^{\mathrm{e}}$ siècle, lorsque le colonel Alfred Dreyfus est accusé à tord de trahison par antisémitisme. De grands écrivains comme Emile Zola prennent alors la parole contre leur gouvernement et l'armée pour défendre l'innocent face au système. C'est ce qui marque, en France, la naissance de cette parole intellectuelle. En ce qui concerne la Pologne, on peut noter que dès le milieu du $\mathrm{XIX}^{\mathrm{e}}$ siècle, les écrivains polonais qui doivent fuir leur pays du fait de leur engagement pour obtenir l'indépendance, comme Adam Mickiewicz qui se fait la voix de la Pologne en France, sont déjà des intellectuels.

L'opinion publique quant à elle se définit comme l'expression du peuple face au pouvoir. Elle se construit à partir de médias, de plus en plus présents et diversifiés au cours $\mathrm{du} \mathrm{XX}^{\mathrm{e}}$ siècle, mais aussi à partir de mouvements plus endogènes, intrinsèques. L'opinion se fonde par des discussions, des rencontres, des représentations d'un événement. Elle a été bien étudiée en France dans les travaux de Robert Franck notamment ${ }^{5}$. Cette opinion publique est en permanente mutation, elle évolue dans le temps. Sa formation gagne avec l'essor de l'éducation; durant la période étudiée, elle peut se construire autour d'arguments que fournissent les intellectuels. En effet, l'intellectuel fait partie des élites, mais ce qui l'en distingue est le fait qu'il se place en médiateur entre le peuple et les élites politiques. Son bagage scientifique lui permet théoriquement de comprendre des ressorts, des concepts qui peuvent apparaitre confus au plus grand nombre; il est censé avoir une

${ }^{3}$ M. B ernard, J. Ła p to s, Les Relations entre la France et la Pologne au XX è s., Eventus, 2002.

${ }^{4}$ On retiendra ici particulièrement ceux de P. Ory, J.-F. S ir in e11i, Les intellectuels en France. De l'affaire Dreyfus à nos jours, col Tempus, éditions Perrin, 2004, mais aussi de M. Tre bits h, M. C. Granjon, dir., Pour une histoire comparée des intellectuels, Complexe, 1996 qui dans son approche comparative permet de montrer que cette définition peut correspondre à ce qui se trouve ailleurs.

${ }^{5}$ R. Frank, «Émotions mondiales, internationales, et transnationales, 1822-1932», Monde $(s), \mathrm{n}^{\circ} 1$, mai 2012, p. 47-70. 
fonction d'interprétation afin d'articuler les différents pôles de la société. Cette capacité acquise d'analyse, de recherche, peut parfois lui donner une vision des choses fondamentalement différente de celle du plus grand nombre, de l'opinion publique. Il est donc intéressant en histoire croisée de montrer en quoi, en étudiant le rapport à un événement étranger de deux acteurs sociaux différents comme les intellectuels et l'opinion publique, s'observe un brouillage des temporalités et de la chronologie. Le phénomène peut être mis en avant dans le cas de la Pologne en France. Parfois, Intellectuels et opinion publique auront la même perception de la Pologne et des Polonais, parfois cette perception sera totalement opposée. Dès lors on observera le rôle des intellectuels pour peser sur l'opinion publique, mais aussi les limites de ces tentatives qui mettent en relief de manière frappante à quel point cette opinion publique se construit de manière complexe.

Pour permettre une meilleure compréhension de ces phénomènes, l'étude prendra en compte dans un premier temps l'arrivée des émigrés en France, qui suscitent les premières réactions des Français, très contrastées avant la Deuxième Guerre mondiale. La période d'après guerre montre comment la trentaine d'années qui suit le conflit est riche en transferts culturels, comment la Pologne trouve une place de valeur en France bien davantage que la Polonia. Enfin l'étude du moment très court et très dense symboliquement du tournant des années 1980, à forte charge émotive en France et en Pologne, permettra de montrer à partir de l'exemple polonais comment l'opinion publique a pu peser sur les intellectuels français.

\section{Les Polonais en France: des images décalées}

Les premiers contacts et donc les premières représentations de la Pologne datent du XVI ${ }^{\mathrm{e}}$ siècle. Le départ d'Henri de Valois pour le trône de France n'empêche par les relations diplomatiques de se développer entre deux pays qui s'imposent dans leur région respective, la France dans l'Europe atlantique et la Pologne en Europe médiane. Déjà, leur point de rapprochement tient à l'Allemagne, que leur position géographique respective circonscrit. L'alliance entre les deux pays semble aller de soi, même si, dans un premier temps, les Français ne cherchent pas à approfondir la connaissance de leur allié. Les échanges diplomatiques voire commerciaux suffisent, comme en témoigne Richelieu dans son Testament politique: «La Pologne et la Moscovie nous sont presqu'inutiles si ce n'est pour réprimer les emprises de l'Empereur; on peut en tirer des fourrures et leur envoyer des étoffes de soie $»^{6}$.

Ces commencements inaugurent des images sur ce pays lointain pour les Français, mais dont les caractères se précisent grâce aux correspondances qui s'échangent dans les milieux nobiliaires. A la cour des rois de Pologne au XVII siècle figurent des nobles français, qui sont les ambassadeurs d'une culture française en expansion à l'Est. La Pologne est décrite dans des lettres envoyées en France, comme celles de la princesse de Gonzague au Grand Condé7. La victoire du roi Jan III Sobieski (1674-1696) sur les Turcs aux portes de Vienne est perçue comme un acte héroïque qui sauve la chrétienté; elle donne

6 D. Tolle t, «Introduction», La Pologne et l'Europe occidentale du Moyen-Âge à nos jours, actes du colloque organisé par l'université Paris VII-Diderot, Paris, 1999, Instytut Historii UAM, Poznań-Paris 2004, p. 8.

${ }^{7}$ Fonds Mazon, Institut d'études slaves. 
à la Pologne pour longtemps cependant une image de "christ des nations", aux répercussions inégales en France. D'autres personnalités polonaises sortent du cadre national polonais pour trouver une place en France, à l'instar de Marie Leszczyńska, femme du roi Louis XV. La cour de son père, le roi déchu Stanislaw Leszczyński, est connue dans l'histoire française pour son rôle dans le soutien aux arts et aux lettres durant le siècle des Lumières. Surnommé «le philosophe bienfaisant», il fait de Lunéville un carrefour culturel, véritable ambassade de Pologne en France, par laquelle passèrent des hommes de lettres, philosophes, militaires, polonais et français.

Ces quelques figures, certes peu nombreuses, suffisent cependant pour marquer les élites françaises, d'autant que nombreux sont les nobles polonais qui circulent alors dans l'espace européen, côtoyant leurs homologues dans les salons. Cette forme de sociabilité est facilitée par le fait que la langue française est devenue, chez les membres de l'aristocratie polonaise, une langue de distinction. Le fait que des Polonais apprennent le français en des temps où la France connait un rayonnement international important est essentiel pour faire entendre la voix polonaise en France. L'écrivain Jan Potocki par exemple, qui fréquente les salons parisiens à la fin du XVIII siècle, témoigne avoir su parler français avant de parler polonais ${ }^{8}$. Cette permanence de la francophonie chez les élites polonaises au XIX ${ }^{\mathrm{e}}$ siècle permet, au moment des insurrections de 1830 et 1848 , à nombre d'entre eux de rejoindre la France pour y trouver refuge'. L'insurrection du 29 novembre 1830 contre le tsar Nicolas $1^{\text {er }}$ à Varsovie provoque une première réaction massive en France, mobilisant l'opinion publique. La répression de la Russie teint d'héroïsme la figure polonaise, héroïsme qui transparait déjà à l'époque napoléonienne avec les hussards polonais. Ressort de ces événements tragiques en Pologne l'image du Polonais face au Russe tel David contre Goliath, luttant contre la férocité de son puissant voisin. Ces images véhiculées en France, qui marquent durablement les esprits et les représentations ultérieures sont construites au sein de l'opinion publique française qui se dissocie à cette occasion de son gouvernement, plus circonspect. Ainsi, le ministre des Affaires étrangères Horace Sébastiani évoque une Pologne «souffrante» mais ajoute qu'il est difficile d'intervenir ${ }^{10}$. Pourtant des pétitions sont signées et des textes appellent à aider des Polonais. Cette résistance qui s'organise depuis la France est entretenue par le regroupement des réfugiés à Paris autour de l'hôtel Lambert acheté par la prince Adam Czartoryski sur l'île SaintLouis. L'élite polonaise qui y est invitée fréquente également les salons parisiens afin de témoigner auprès des élites françaises de la situation d'un pays encore peu connu. Le salon de Marie d'Agoult, celui de Frédéric Ozanam figurent parmi ces lieux de médiation entre les deux cultures au XIX ${ }^{e}$ siècle $^{11}$. Ces liens permettent la création de la Bibliothèque

8 Z. Marki ew ic z, « Jean Potocki, auteur du Manuscrit trouvé à Saragosse, et ses liens avec les intellectuels français des Lumières ", in Approches des Lumières: mélanges offerts à Jean Fabre, éd. Klincksieck, Paris, 1974, p. 309.

9 Voir notamment L"Autre Francophonie, sous la dir. de J. Nowicki et C. Mayaux, éd. Honoré Champion, 2012.

10 D. Beauvois, «L'accueil des Polonais en France après l'insurrection de 1830-1831 », in Polonia, des Polonais en France de 1830 à nos jours, ,sous la direction de Janine Ponty, Montag, 2010, p. 13.

${ }^{11}$ De nombreux articles à ce sujet figurent dans l'ouvrage collectif Contacts religieux franco-polonais : du Moyen Âge à nos jours: relations, influences, images d'un pays vu par l'autre: colloque international, Lille, 5-7 octobre 1981, Éditions du dialogue, CNRS, 1985. 
polonaise, qui a pour vocation de maintenir vivante la culture polonaise au-delà de ses frontières ${ }^{12}$. Néanmoins, ceux que l'on surnomme la «Grande Emigration» marquent surtout les représentations des intellectuels français ; l'opinion française pour sa part construit sa vision de la Pologne à partir d'autres référents. Et comme pour chaque événement qui peut soulever une émotion collective et mobiliser, le temps fait retomber assez rapidement les engagements menés. Le contexte des relations internationales et l'évolution de la vie politique française à la fin XIX ${ }^{\mathrm{e}}$ siècle détourne l'attention de la Pologne, au profit de la Russie. Dès lors il faut attendre les années 1920/1930 pour voir à nouveau la Pologne devenir un enjeu en France.

En effet, la renaissance de la Pologne après la Première Guerre mondiale s'accompagne du soutien des autorités françaises, qui très vite signent des accords de coopération aux ressorts multiples, à la fois défensifs, économiques et culturels, qui affirment la nécessité de contacts entre nouvelles élites polonaises et les Français. Les accords de coopération scientifiques et culturels donnent les moyens financiers aux chercheurs, aux intellectuels d'aller en France ou en Pologne, et d'intervenir au sein des universités. La création de l'Institut français de Varsovie est un des aspects de la volonté de maintenir la présence française en Pologne, et d'avoir un lien particulier avec ce pays. Dès lors effectivement, des échanges se développent d'un point de vue universitaire, par exemple en histoire avec la IV section de l'EPHE (École Pratique des Hautes Études), l'école des Annales ou encore en linguistique, avec le rôle de l'Institut d'études slaves en France; les transferts de connaissance et les influences se multiplient ${ }^{13}$. De nombreux étudiants par ailleurs viennent à Paris ${ }^{14}$. C'est donc surtout dans les milieux scientifiques que les contacts se développent tout comme des relations solides. Une partie des intellectuels français cependant se montre de plus en plus circonspecte à l'égard de la Pologne. La naissance de l'URSS est perçue de manière positive par nombre d'entre eux; de fait, comme à de nombreuses occasions dans l'histoire, le rapprochement avec la Russie/Union soviétique suppose un éloignement de la Pologne en France. Cette dernière apparait comme agressive ${ }^{15}$. Ces intellectuels attirés par la «Grande lueur vers l'Est» sont encore très minoritaires dans le monde intellectuel français. Ils sont en revanche très proches dans leur hostilité à la Pologne d'une grande partie de l'opinion française.

En effet, au niveau de la majorité de la population française et en dehors de Paris, les contacts avec la Pologne se font par le biais des émigrés polonais qui arrivent en France dans le cadre des relations économiques. En effet, dès 1919, la France signe des conventions pour employer une main d'œuvre étrangère. Le choix de la Pologne se justifie par son statut d'allié. D'autres facteurs agissent en ce sens, ils ont été mis en valeur par l'historienne Janine Ponty, qui en a bien analysé ces processus. Les Polonais sont choisis pour

${ }^{12}$ D. Dubois, «La Société historique et littéraire polonaise et la Biblioteka Polska de Paris », in Polonia..., p. 30.

${ }^{13} \mathrm{M}$. Will a u me, «La présence des sciences humaines polonaises en France entre-les-deux-guerres », in Les relations entre la France et la Pologne au XXe siècle, op. cit., p. 28; M. Tr e bits c h, «La seconde patrie: l'accueil des intellectuels d'Europe centrale et orientale à Paris dans l'entre-deux-guerres », in Paris, "capitale culturelle» de l'Europe centrale?, sous la dir. de M. Delaperrière, Institut d'Etudes slaves, 1997, p. 29.

${ }^{14} \mathrm{~A}$. M a rè s, «Aperçu des communautés centre-européennes à Paris dans l'entre-deux-guerres», in Paris, «capitale culturelle» de l'Europe centrale?, p. 20.

15 Voir notamment les article de l'Europe nouvelle comme "Les fautes de la Pologne", daté du 18/07/1920. 
leur réputation de travailleurs mais aussi par l'écho que donne l'intelligentsia parisienne au surpeuplement des campagnes polonaises et à la misère qui règne dans les mines de Haute-Silésie. Les contrastes sociaux qui se révèlent après la guerre montrent le dénuement, surtout à l'ouest du pays, dans lequel se retrouvent une forte proportion d'ouvriers agricoles. Comme le montre Janine Ponty à partir d'archives diverses, le gouvernement polonais, dans sa volonté de moderniser le pays, se retrouve face à la problématique d'un pays trop agricole, en retard sur l'industrialisation. Dès lors le départ d'une bonne partie de cette main-d'œuvre vers la France est une soupape salutaire pour permettre les réformes ${ }^{16}$. Les Polonais recrutés après de nombreuses procédures administratives sont répartis dans toute la France. Ils sont sous contrat dans des fermes ou des mines, en priorité dans des régions françaises de grande culture dévastées par la guerre, comme en Picardie, en Lorraine. Les Français qui font appel à de la main-d'œuvre polonaise n'ont pas du tout les mêmes références que les élites: il s'agit surtout de trouver des travailleurs solides et endurants: «Tant qu'à prendre des ouvriers qui ne comprenaient pas notre langue, autant choisir ceux habitués à nos climats $»^{17}$. L'intégration des Polonais est d'autant plus difficile qu'elle n'est pas souhaitée par la France dans les années 1920. Les migrants sont perçus avec défiance et victimes de mesures arbitraires : l'étranger effraie. Tout au long des années 1920, il y a deux civilisations et deux conceptions de la migration qui se heurtent. Comme le dit Janine Ponty, les «immigrés s'accrochent, comme un moindre mal, à un pays qui semble ne plus vouloir d'eux $»^{18}$. En 1927 , les frontières sont fermées à la main-d'œuvre étrangère, ce qui va dans le sens de ce que souhaite l'opinion publique française. La crise de 1929 accentue les tensions sociales et pousse le gouvernement français à organiser des rapatriements. Janine Ponty caractérise ainsi cette réception des Polonais en France:

A l'exception d'une courte parenthèse lors du Front populaire, le rejet l'emporte sur l'acceptation dans l'esprit des Français et les Polonais ont la vie dure. De 1920 à 1930, ils choquent et ils étonnent. De 1931 à 1936, ils étonnent moins mais ils gênent. De 1938 à la guerre, ils déplaisent ${ }^{19}$.

Au seuil de la Deuxième Guerre, l'opinion française est donc globalement plutôt défavorable à la Pologne et aux Polonais. Sentiment qui va se poursuivre bien au-delà du conflit.

\section{L'après 1945: le rôle du progressisme dans l'image de la Pologne en France}

La fin de la Deuxième Guerre mondiale ne modifie pas l'image des Polonais présents en France, au contraire. Pendant longtemps, particulièrement dans les campagnes françaises, l'image des immigrés polonais sera ternie par l'idée que la France est entrée en guerre à cause de Gdansk et de la Pologne. De nombreux Polonais doivent justifier de leur présence en France et sont victimes d'une discrimination sourde, qui passe par des termes péjoratifs tels que «Polaks» et par une volonté dans les petits villages de leur refuser toute

${ }^{16}$ J. P o n ty, Polonais méconnus, Histoire des travailleurs immigrés en France dans l'entre-deux-guerres, Publications de la Sorbonne, 2005, p. 44.

${ }^{17}$ Ibidem, p. 93.

18 Ibidem, p. 220.

19 Ibidem. p. 388. 
évolution sociale. Ces conditions et le fait que nombre d'entre eux aient toujours espéré retourner dans leur pays font que, entre 1946 et 1948, beaucoup cèdent à la propagande communiste et retournent en Pologne ${ }^{20}$. Du côté de l'opinion publique française, c'est moins la situation à l'Est et l'immigration qui importe que l'enjeu de la décolonisation et des conflits qui éclatent en Indochine puis en Algérie.

Cette distance à la Pologne et aux Polonais en France se constate également du point de vue des intellectuels français. Ces derniers sortent de la Deuxième Guerre mondiale sous l'influence du Parti communiste français, qui incarne pour nombre d'entre eux une résistance courageuse et difficile contre l'ennemi. Pour les Français, c'est l'URSS qui a permis la victoire. De nombreux intellectuels français se mettent à militer dans le PCF (Parti Communiste français); d'autres, tel Jean-Paul Sartre, deviennent des compagnons de route : sans adhérer, ils alimentent et soutiennent son discours. Cette posture caractérise particulièrement les intellectuels progressistes, qu'ils soient inscrits au Parti, animant ses grandes revues comme Les Lettres françaises, La Nouvelle Critique, ou des compagnons de route comme les Temps Modernes ou encore la revue Esprit ${ }^{21}$. Ces progressistes défendent l'URSS et refusent de considérer le système soviétique comme un système totalitaire ${ }^{22}$. Ils souhaitent poursuivre la lutte antifasciste contre les Américains qui prennent la place des Allemands dans leurs discours ${ }^{23}$. La Pologne communiste qui se construit alors incarne pour nombre d'entre eux une terre nouvelle, où il va être possible de reconstruire sur de nouveaux fondements susceptibles de garantir la paix. Car c'est autour de cette idée que l'URSS rencontre l'adhésion de nombre d'intellectuels. Ainsi, Frédéric Joliot-Curie, gendre de Marie Curie, intervient en Pologne en janvier 1947 pour inciter les scientifiques à s'engager, à quitter leur tour d'ivoire pour la paix au nom de leur responsabilité dans la création d'inventions néfastes pour l'homme ${ }^{24}$. Nombreux sont les intellectuels français qui se déplacent en 1948 pour participer au Congrès de la paix organisé à Wrocław ${ }^{25}$. Paul Éluard, Pierre Daix, Pablo Picasso, Julien Benda, Vercors, Roger Vailland, Pierre Seghers Aimé Césaire, Yves Farge, l'abbé Boulier, Marcel Prenant, ou Fernand Léger font le déplacement. La Pologne détruite que les autorités communistes ne manquent pas de leur faire visiter alors renforce encore plus leur adhésion au camp de la paix qui se trouve, selon eux, à l'Est. Dès lors tous ceux qui voudraient s'opposer au communisme sont rejetés dans le camp «fasciste», d'une manière assez caricaturale mais qui apparait alors nécessaire.

Or les voix s'opposant aux modalités de gouvernement qui se mettent en place en Pologne après la guerre ne manquent pas de la part d'intellectuels polonais qui se retrouvent en France après 1945, notamment des soldats de l'armée de Anders. Certains d'entre eux, tels Józef Czapski, Kazimierz Romanowicz, Jerzy Giedroyc et Zygmunt Hertz ont l'ini-

20 J. P onty, Les Polonais en France, Editions du Rocher, 2008, p. 173.

${ }^{21}$ P. Ory, Jean-François Sirine 11 i, Les intellectuels en France. De l'affaire Dreyfus à nos jours, p. 257.

22 P. Grémion, "Voix d'exil en marge du progressisme parisien», in Intellectuels de l'Est exilés en France, sous la dir. de W. Falkowski et A. Marès, Institut d'études slaves, Paris, 2011, p. 33.

23 P. Ory, J.-F. S irin el1i, Les intellectuels en France, p. 280.

24 M. P a s z tor et D. J a ros z, Conflits brulants de la guerre froide: les relations franco-polonaises de 1945 à 1954, chez Lavauzelle, 2005, p. 85.

25 J. Łaptos, «Le pacifisme apprivoisé: le congrès des intellectuels pour la défense de la paix en 1948 », in Le pacifisme en Europe des années 20 aux années 1950, sous la direction de Maurice Vaïsse, édition Bruylant, 1993, p. 325. 
tiative de créer une librairie polonaise sur l'île Saint Louis afin de reconstituer un centre intellectuel polonais. Tout comme la Bibliothèque polonaise qui, en son temps, avait été créée afin de préserver une culture en danger, la librairie libella se veut un mode d'action contre l'occupation soviétique. Très vite, elle devient la cible des attaques de la propagande communiste en France.

Un article de l'Humanité daté du 3 avril 1947 dénonce la présence d'un état-major « illégal» à l'hôtel Lambert, où sont livrés «d'énormes caissons qui contiennent sans doute autre chose que les archives personnelles du commandant Czapski ${ }^{26}$. L'article cherche à diffamer la présence de ces soldats polonais en suggérant leur collusion avec les Allemands. La librairie Libella reçoit des tracts qui réclament son départ: «Dehors les fascistes de l'île Saint-Louis $\gg{ }^{27}$. Ces attaques violentes qui occultent l'héroïsme de ces soldats sont un des éléments qui expliquent la volonté de Jerzy Giedroyc et de son équipe de s'installer un peu à l'écart de Paris, à Maisons-Laffitte, pour poursuivre leur travail éditorial. Et c'est en effet loin des centres de la vie intellectuelle française que se développe une opposition au régime communiste en Pologne depuis la France.

La distance qui se crée entre Polonais et Français en France après la guerre ne suppose pas cependant un éloignement de la Pologne en France, du moins du côté des intellectuels. En effet, ce qui les intéresse à présent est moins le discours que peuvent véhiculer sur leur pays les Polonais émigrés en France que ceux qui sont restés sur place. Néanmoins les possibilités de se rendre en Pologne, ou de faire venir des Polonais en France sont très réduites entre 1949 et 1953, durant la période stalinienne. Les relations diplomatiques sont également interrompues. Il faut attendre 1955 pour que les premiers rapprochements aient lieu, faisant d'ailleurs intervenir des intellectuels français et polonais, principaux acteurs de la «diplomatie culturelle». Dans le cas de la Pologne, ces médiateurs sont des personnes qui avaient tissé des liens d'amitié avec leurs homologues polonais, comme Michel Lejeune, directeur adjoint au CNRS (Centre National de la Recherche scientifique). D'autres comme Jean Fabre avaient vécu en Pologne avant la guerre et appris le polonais ${ }^{28}$. Avec les encouragements du Quai d'Orsay, certains universitaires français s'organisent pour permettre le rapprochement franco-polonais, qui aboutit en septembre 1956 à une rencontre entre des représentants éminents des deux pays. Les intellectuels polonais qui viennent en France sont invités à participer à des séminaires en Sorbonne sur le thème du «progrès social et économique ${ }^{29}$, avec des intervenants comme Fernand Braudel, Georges Friedmann, Claude Lévi-Strauss, Charles Morazé ou encore Ernest Labrousse. Il s'agit d'une rencontre scientifique avant tout, qui multiplie les approches disciplinaires et confronte l'élite intellectuelle des deux pays ${ }^{30}$.

${ }^{26}$ L'Humanité, 3 avril 1947.

27 Libella Galerie Lambert, Uniwersytet Mikołaja Kopernika, Toruń, p. 172.

${ }_{28}$ Pour davantage d'informations voir notre article sur «Jean Fabre : une figure emblématique des relations intellectuelles franco-polonaises», in La France et l'Europe centrale, médiateurs et médiations, sous la dir. d'A. Mares, Institut d'études slaves, Paris, 2015.

${ }^{29} \mathrm{~L} . \mathrm{Ku} \mathrm{k}$, «Les échanges culturels et scientifiques franco-polonais à l'épreuve de la guerre froide », Une Europe malgré tout, 1945-1990. Contacts et réseaux culturels, intellectuels et scientifiques dans la guerre froide, sous la direction d'A. Fleury et L. Jilek, édition Peter Lang, p. 448.

${ }^{30}$ M. Frybes, «L'Octobre polonais et ses conséquences pour les relations franco-polonaises », in 1956, une date européenne, sous la direction de G. Mink, M. Lazar et M. J. Sielski, Éditions Noir sur Blanc, 2010, p. 255 . 
En effet, pour ce moment solennel qui doit marquer la reprise des relations officielles entre les deux pays, la Pologne envoie à Paris un panel de scientifiques reconnus pour leurs travaux dans différents domaines: sociologie (Nina Assorodobraj-Kula), philosophie (Bronisław Baczko et Leszek Kołakowski), histoire (Alexandre Gieysztor et Witold Kula), économie (Edward Lipiński) ou droit (Edmund Cieślak). La rencontre est un tel succès qu'un an plus tard, des accords de coopération scientifique sont signés entre la PAN (Académie des sciences polonaise) et le CNRS d'une part, et la PAN et la sixième section de l'EPHE d'autre part. C'est Fernand Braudel qui signe du côté français ; ce dernier entame ainsi une longue correspondance avec les historiens polonais, comme le montrent ses archives personnelles. Côté polonais, c'est surtout l'historien Tadeusz Manteuffel qui œuvre dans le sens de ces rapprochements ${ }^{31}$.

Ces accords, a priori très Théoriques, sont devenus néanmoins essentiels pour permettre les contacts. Dans le contexte de la division de l'Europe en deux blocs, les relations scientifiques sont perçues de manière favorable et ne sont pas sujet de tension. Les accords permettent un financement des voyages, dans un contexte où le différentiel de revenus entre la France et la Pologne devient important. Pour les chercheurs polonais, les bourses et les financements sont bienvenus. Pour les Français également, mais dans une mesure bien différente. Grâce à ces accords, de nombreux chercheurs polonais viennent en France d'une part pour approfondir leurs recherches, et d'autre part pour montrer les réussites de la science polonaise de l'après-guerre. En revanche les scientifiques français qui participent à ces échanges témoignent de leur curiosité et de leur volonté de mieux connaitre le bloc communiste. Des exemples bien connus comme celui de Jacques le Goff permettent de montrer comment l'amitié qui se crée entre chercheurs, en l'occurrence ici avec Bronisław Geremek dès 1959, permettent aux Français d'approcher les milieux intellectuels polonais ${ }^{32}$. D'autres encore se rendent en Pologne grâce aux accords, surtout dans les années 1960. A chaque fois, c'est autant d'occasions de rencontres, d'autant que la Pologne est le principal pays du bloc Est avec lequel les accords fonctionnent.

En dehors des accords, d'autres liens se construisent après 1956 dans le cadre de l'arrivée au pouvoir de Gomułka qui attire les regards des intellectuels français jusque-là peu prolixes au sujet de la Pologne. Si les intellectuels français s'expriment davantage sur les événements de Hongrie et leur violence, les propositions de changement portées par les intellectuels polonais ne manquent pas de les interpeller. Á partir de 1957, certains intellectuels observent et tentent d'interpréter, sinon de projeter en France, les changements en cours en Pologne. Différentes revues proposent un éclairage sur ce que les Français interprètent comme un nouveau type de socialisme émergent en Pologne, qui prend le nom ambigu de «révisionnisme». Pour les intellectuels français, le terme a une forte charge positive, puisqu'il propose, selon eux, une autre option que le stalinisme. Pour étayer cette proposition, des revues comme Arguments, Les Temps Modernes ou Esprit proposent des articles qui donnent la parole aux Polonais ${ }^{33}$. Au-delà de la Pologne, c'est toute une réflexion autour de la Gauche que les intellectuels tentent de reconstruire. Durant la deu-

${ }^{31}$ Lidwine Warchol, La Pologne en France, les relations entre intellectuels français et intellectuels polonais de 1966 à 1983, thèse de doctorat soutenue en décembre 2014 sous la direction d'Antoine Mares, Panthéon Sorbonne, p. 90.

32 Ibidem, p. 111.

${ }^{33}$ Voir notamment le numéro de Temps Modernes de janvier 1957, le numéro de mai-juin 1958. 
xième moitié des années 1960, alors que les intellectuels polonais dits «révisionnistes» entrent peu à peu en conflit avec le pouvoir, des transferts ont lieu vers la France, notamment celui de la lettre ouverte au Parti rédigée par Jacek Kuroń et Karol Modzelewski. Les modalités de ce transfert ont été étudiées dans le détail par Marta Kirszenbaum ${ }^{34}$. Ce transfert alimente une réflexion commune entre la France et la Pologne, alors que de nombreux intellectuels français commencent à s'éloigner du Parti communiste français tout en cherchant une voie d'engagement à gauche. C'est dans ce contexte que certains d'entre eux adhèrent dans les années 1960 au PSU, le Parti socialiste unifié. Ce sont les intellectuels français proches de ce mouvement de gauche qui sont, à partir de cette date, en relation de plus en plus étroite avec les intellectuels polonais, comme Jean-Marie Domenach et les rédacteurs d'Esprit ${ }^{35}$, mais aussi Ernest Labrousse ${ }^{36}$, Jacques Le Goff ${ }^{37}$ ou François Furet. Il y a l'espoir de créer un nouveau socialisme dont l'exemple viendrait notamment de Pologne ${ }^{38}$.

La Pologne en France apparait donc, à la fin des années 1960, du moins dans l'optique des intellectuels français sous un angle nouveau. L'image traditionnelle d'une Pologne avant tout incarnée par son catholicisme et sa lutte désespérée contre des puissants laisse place dans ces milieux à une Pologne vivier d'une proposition politique nouvelle, émanant de scientifiques reconnus et respectés par la communauté internationale. Les voyages scientifiques ont montré en effet aux Français à quel point les Polonais ont acquis un haut degré de compétences dans tous les domaines, autant dans les sciences dites «dures» que dans les sciences humaines ${ }^{39}$. Dès lors, les discours des intellectuels polonais connaissent une réception nouvelle en France. Dans le contexte de la recomposition de l'opposition polonaise au début des années 1970, cette perception va être précieuse pour les intellectuels polonais. Les accords d'Helsinki signés en 1975 permettent l'expression des oppositions politiques dans les pays du bloc soviétique. La thématique des droits de l'homme, acceptée par l'URSS et ses satellites au nom de la Détente, offre une brèche essentielle pour faire pression sur les gouvernements communistes. En échange de la reconnaissance de ses acquisitions territoriales et de son influence sur les pays de l'Est, les pays de l'Alliance atlantique demandent le respect des droits de l'homme à l'intérieur de chaque État. Si ces accords sont très critiqués, il n'empêche qu'il s'agit aussi officieusement d'encourager, sous couvert des droits de l'homme, la contestation

${ }^{34}$ M. K irsze n ba u m, 1968 de Varsovie à Paris : transferts, réceptions et occurrences du mouvement étudiant polonais dans la contestation universitaire parisienne, IEP, Master 2, sous la dir d'Emmanuelle Loyer, sept. 2008, et «1968 entre Varsovie et Paris: un cas de transfert culturel de contestation», Histoire@Politique. Politique, culture, société, $N^{\circ}$ 6, septembre-décembre 2008, ou encore il est possible de se référer à «De Varsovie à Paris : réceptions, influences et occurrences du mouvement étudiant polonais dans la contestation universitaire parisienne », in J. F a u re, D. Roll and (dir.), 1968 hors de France: histoire et constructions historiographiques, L'harmattan, 2009.

35 G. B ou d i c, Esprit 1944-1982, Les métamorphoses d'une revue, éditions de l'IMEC, 2005, p. 222.

${ }^{36} \mathrm{~V}$. Duclerc, «La 〈deuxième gauche〉», in Histoire des gauches en France. Volume 2., XX $X^{e}$ siècle, à l'épreuve de l'histoire, sous la dir. de Becker Jean-Jacques et Gilles Candar, La Découverte, 2005. p. 184.

${ }_{37} \mathrm{~J}$. Le Goff, Une vie pour l'histoire, op. cit., p. 71.

${ }_{38}$ M. Winock, «La gauche non communiste en France: la coupure de 1956 », issu de l'ouvrage 1956 Varsovie-Budapest, La deuxième Révolution d'Octobre, Textes réunis par P. Kende et K. Pomian, collection Esprit/Seuil, 1978, p. 147.

39 Témoignage du physicien Yves Quéré, ou encore de Jacques le Goff. 
contre l'URSS ${ }^{40}$ : les occidentaux obtiennent que l'Acte final soit diffusé dans chaque pays du bloc.

C'est cette thématique que vont utiliser les intellectuels polonais au moment où éclatent, le 25 juin 1976, des grèves dans les usines d'Ursus et de Radom. Signalées par les principaux quotidiens français, elles sont décrites selon la terminologie du gouvernement polonais à savoir des «révoltes» très vite maîtrisées par le pouvoir. Elles avaient donc vocation à rester «silencieuses » en France à l'instar des mouvements de 1970 qui ont reçu très peu d'échos en France ${ }^{41}$. Or c'est la mobilisation des intellectuels polonais aux côtés des ouvriers pour obtenir des droits et une amélioration de leur situation qui va enclencher une réaction de la part des intellectuels français. En effet, l'étude des quotidiens français montre que les événements polonais rencontraient peu d'intérêt en France. Cela peut s'expliquer par le fait que les relations entre le gouvernement polonais, incarné par la personne d'Edward Gierek, avec le président français Valéry Giscard d'Estaing sont très étroites. Dans les médias français, Edward Gierek est présenté depuis le début des années 1970 sous un jour très favorable; il ne semble pas imaginable qu'une critique soit possible en France. Ces relations favorables ont donc l'inconvénient de limiter l'écho des voix polonaises qui veulent critiquer le pouvoir communiste et démocratiser le système. En revanche, elles ont l'avantage de devenir un vecteur pour peser sur le gouvernement polonais, très dépendant de l'opinion internationale et qui souhaite faire bonne figure en France. Par un système complexe de relais, de médiateurs polonais en France, la révolte ouvrière trouve un soutien énorme à partir de novembre 1976 parmi les intellectuels français. Le KOR et certaines figures tutélaires comme Jacek Kuroń, Adam Michnik trouvent un écho en France et braquent les projecteurs des médias français sur une Pologne dont on ne parlait plus. Des intellectuels comme Jean-Paul Sartre s'investissent, des revues comme le Nouvel Observateur, le Monde parlent pendant plusieurs mois très régulièrement de l'évolution de la situation polonaise ${ }^{42}$. Cet engouement de la part des intellectuels français s'explique par ce que représente pour eux cette alliance que propose le KOR entre intellectuels et ouvriers, à savoir un modèle de socialisme davantage autogestionnaire que dirigé. Il s'agit donc de questionner leurs propres représentants politiques, tiraillés en 1977 entre François Mitterrand qui accède à la tête du parti socialiste alors que beaucoup appelaient de leurs vœux l'élection de Michel Rocard, de la Deuxième Gauche qui peu à peu prend le surnom de "Gauche polonaise». Des intellectuels d'origine polonaise comme K.S Karol, Georges Mink, Krzysztof Pomian traduisent par leurs articles et leurs interventions une situation polonaise difficile à comprendre du côté français: ces transferts ne sont possibles, surtout dans les milieux intellectuels, que par des traducteurs dont le statut est reconnu pour permettre l'engagement. La démarche porte ses fruits: tous les opposants et les ouvriers prisonniers sont libérés par une amnistie le 22 juillet 1977 . Le pouvoir polonais a décidé de reculer face à une pression qui s'exerce non seulement en Pologne, mais aussi au-delà, parmi les opinions publiques de ses créanciers. Durant toute cette période l'investissement des intellectuels français a été le plus intense. Mais dès septembre 1977 l'engouement-sinon l'engagement retombe.

\footnotetext{
40 J. A n d ré a n i, Le piège, Helsinki et le monde communiste, Odile Jacob, 2005, p. 48.

${ }^{41}$ Lidwine Warc h o 1, La Pologne en France..., p. 222 et suiv.

${ }^{42}$ Ibidem, p. 230 et suivantes.
} 


\section{Le tournant des années 1980 : les enjeux de la Pologne en France?}

De 1977 à la déclaration de l'état de siège le 13 décembre 1981, les intellectuels français vont en effet prendre une certaine distance avec la Pologne en France, voire tenter de minimiser cette présence. Les articles publiés en France dans les principaux quotidiens français pour soutenir l'opposition polonaise tout au long de l'année 1977 ont analysé les limites des régimes dits «socialistes» à l'Est, et fortement attaqué les partis communistes. Or, en 1972, le parti socialiste français et le parti communiste ont signé une alliance appelée «Union de la gauche», qui doit conduire à une victoire électorale très attendue par une majorité des intellectuels français. L'implication de nombre d'entre eux, avec des arguments solides et étayés, des témoignages sur ce que peut être le «socialisme réel», tend à décrédibiliser dans l'opinion publique le Parti communiste français lui-même et, plus largement, met en péril l'Union de la gauche ${ }^{43}$. Cet aspect apparait nettement à la lecture des critiques qu'émettent des militants de gauche dans les colonnes de certaines revues militantes comme Le Nouvel Observateur. Ainsi, le rédacteur en chef Jean Daniel se voit reprocher par ses lecteurs son manque de soutien à l'Union de la gauche. Ce dernier tente de justifier la ligne de son journal par l'idée que «l'enjeu idéologique est essentiel » ${ }^{44}$. Pour lui, les régimes communistes proposent un modèle biaisé : «Il y a quelque chose de pire que le capitalisme c'est le capitalisme d'État ${ }^{45}$. Au même moment, le PCF rompt l'Union de la gauche, ce qui constitue un choc brutal pour nombre de militants socialistes, à quelques mois des élections législatives en France. Beaucoup se rendent compte de la difficulté de transférer des luttes politiques de l'étranger en évitant les amalgames. Pour la population française, critiquer le «socialisme» à l'Est, en montrer les aspects totalitaires et répressifs, c'est remettre en cause le socialisme en général, particulièrement en France. Or les intellectuels français dans leur grande majorité sont encore plus hostiles à Valéry Giscard d'Estaing qu'à Georges Marchais, dont ils avaient pourtant accepté l'alliance. C'est pourquoi septembre 1977 marque un tournant majeur dans le monde intellectuel français dans le rapport à la Pologne: le combat des dissidents ne doit plus avoir d'écho pour éviter d'influer sur le champ politique français.

Cette mise à l'écart assez générale dans le monde intellectuel français de la Pologne n'est en revanche pas du tout représentative de l'évolution de l'image de la Pologne parmi l'opinion publique française. Il se trouve que la fin des années 1970 correspond à une nouvelle sensibilité autour de la présence de l'immigration polonaise en France. Il s'agit d'un autre sujet de recherche en soit, qui ne peut figurer ici. L'hypothèse qui peut être avancée cependant étant que la France, à partir de 1945, a connu d'autres vagues d'immigration qui ont fait rejeter sur d'autres toutes les représentations négatives, tous les clichés que les Polonais avaient eux-mêmes vécus, phénomène récurrent en histoire. Une intégration s'opère, la perception de cette population se modifie. Ce changement, d'après les témoignages recueillis, est particulièrement perceptible à partir de l'élection du pape polonais Jean-Paul II en 1978. Le personnage bénéficie d'une image très positive dans la population française; son dynamisme, sa prestance, son charisme est reconnu de tous. Adulé par une grande partie

${ }^{43}$ Ibidem, p. 286.

${ }^{44}$ Jean D a n i e l, éditorial, Le Nouvel Observateur, semaine du 26 septembre 1977.

${ }^{45}$ Ibidem. 
des Polonais de France, il donne une nouvelle image de la Pologne à travers le monde, à la fois ferme et à la fois rassurante avec la phrase «N'ayez pas peur», largement reprise dans les médias ${ }^{46}$. Cette image positive doit être très nuancée en ce qui concerne les intellectuels français. Le rapport au pape est un des aspects qui montre comment s'accentue, à la fin des années 1978, une distance entre les intellectuels français et leur opinion publique qui montre aussi une volonté «autogestionnaire» de la population française, à savoir la volonté de construire une opinion indépendante de ce que peuvent en dire différents médias.

En effet, les archives de Jerzy Turowicz, par exemple, montrent que si de nombreux correspondants français, des intellectuels catholiques, sont heureux de cette élection, ils s'interrogent quant à la forme de gouvernement religieux que Jean-Paul $\mathrm{II}^{47}$. Des universitaires comme François Chirpaz, ou Paulette Mounier ${ }^{48}$ expriment leur « joie». Cette dernière voit dans cette élection la promotion d'un peuple profondément croyant, qui incarne une certaine forme de catholicisme reflet d'un effort tenace pour maintenir une religion ouverte. L'ancien rédacteur en chef de la revue Esprit, Jean-Marie Domenach exprime sa satisfaction après l'élection du pape dans un courrier personnel adressé à Tadeusz Mazowiecki ${ }^{49}$. Beaucoup se rendent en Pologne, à Cracovie ou encore à Lublin, invités par la KUL tels les historiens Jean-Marie Mayeur et René Rémond ${ }^{50}$.

D'autres intellectuels français se montrent favorables tout en restant plus distanciés: Louis de Villefosse dans un article de Contrepoints interroge l'élection du pape et la «question»du catholicisme polonais ${ }^{51}$. Évoquant l'histoire du «peuple martyr», Villefosse doute de la compatibilité du catholicisme polonais avec les aspirations de l'Occident libéral. Néanmoins le rôle que tient l'Église polonaise auprès de l'opposition et du KOR agit, parmi ces milieux intellectuels, comme un élément favorable. Les intellectuels français en effet, grâce aux articles des intellectuels polonais que sont Adam Michnik ou Leszek Kołakowski, ont compris le rôle de l'Eglise en Pologne et toute la subtilité de son combat contre les communistes. Il a fallu néanmoins ce transfert habile de la part de personnalités remarquables et respectées pour que les intellectuels français puissent le comprendre. Le pape Jean-Paul II lui-même est toujours présenté en France, à la fin des années 1970, dans son rôle à Cracovie aux côtés des dissidents et dans sa proximité avec la plupart des intellectuels polonais.

Mais ce rôle, dans le contexte de l'enjeu électoral en France, tend à être minimisé pour favoriser une réussite politique. Encore une fois, l'élection de Jean-Paul II présenté dans tous les médias comme «le vicaire de la dissidence $»^{52}$, met en accusation les régimes communistes qui se retrouvent confrontés désormais à un adversaire de taille. Pour certains intellectuels français foncièrement hostiles à l'égard des excès que peut comporter le religieux, une défiance apparait. Nombre d'entre eux tentent alors de minimiser l'impact

${ }^{46}$ De nombreuses biographies de Jean-Paul II insistent sur cet aspect en France, notamment celles de B. L e c o m t e, La vérité l'emportera toujours sur le mensonge, Paris, J. C. Lattès, 1991.

47 Bibliothèque polonaise de Paris, archives Turowicz, correspondance, lettre du 16 octobre 1978.

${ }^{48}$ Paulette Mounier est la femme d'Emmanuel Mounier, le fondateur de la revue Esprit

49 IMEC (Institut Mémoires de l'édition contemporaine), correspondance Domenach, lettre du 17 janvier 1979 , dossier $2 / 2$.

${ }^{50}$ Entretien de Jean-Marie Mayeur avec l'auteur.

${ }^{51}$ Louis de Ville fo s s e, «Réponse à Jean-Paul II », Contrepoints n 29, 1979, p. 39.

52 Jean Da ni e l, éditorial du Nouvel Observateur daté du 2 juin 1980. 
de l'élection du pape afin de limiter les transferts des événements polonais en France. Il faut toute l'habilité d'intellectuels médiateurs comme Krzysztof Pomian pour réussir à stopper les arguments de certains intellectuels français. Par exemple, dans un entretien publié dans la revue intellectuelle de gauche Faire, qui jusque-là parle peu de la Pologne, Krzysztof Pomian replace le rôle du pape dans une histoire de la dissidence, tout en le replaçant bien dans sa fonction religieuse. Face aux inquiétudes exprimées par la revue, il parvient à replacer l'importance de la contestation polonaise, en montrant que la Pologne, présentée parfois comme «rétrograde» en France, est particulièrement en avance en ce qui concerne l'alliance entre les ouvriers avec les intellectuels dans le but de placer le pouvoir face à ses responsabilités ${ }^{53}$.

A la fin des années 1970, les opinions sont donc multiples en ce qui concerne la Pologne. Malgré une certaine volonté des milieux politiques et intellectuels de minimiser la voix de la dissidence à l'Est, les événements réactivent par d'autres biais la présence de la Pologne en France. L'intérêt de la population française se renforce pour ce pays qui apparait à nouveau comme «martyr», opprimé par l'URSS. De nombreux individus vont prendre le contrepieds de l'image que certains intellectuels français tentent de diffuser sur la Pologne. Ainsi, les archives de Lucienne Rey, journaliste et écrivain polonaise vivant en France, montrent qu'elle conteste régulièrement la vision que certains articles tentent de donner sur la Pologne. Par exemple, Lucienne Rey écrit à Guy Lemonnier, qui fait paraître ses articles dans la revue Est et Ouest, pour protester contre les modifications apportées à l'un de ses articles. Il s'agit d'y présenter les Polonais comme « des gros consommateurs de viande $\aleph^{54}$. Elle rectifie en rappelant la pénurie qui sévit en Pologne, mais dont visiblement les intellectuels français qui interviennent à ce sujet n'ont pas du tout pris la mesure : c'est comme s'il était difficile d'accepter le degré de délabrement auquel a conduit le système socialiste en Pologne. Cette attitude laisse à penser que reconnaître la terrible situation économique polonaise pour les intellectuels français revient à les accuser. Les événements des années 1980 en Pologne affectent brutalement l'image de superpuissance incarnée par l'URSS, écornée par l'image de la situation économique désastreuse à l'Est.

Cela dit, nombreux sont ceux qui savent la réalité de la situation et qui tentent d'aider concrètement les Polonais, montrant à quel point il $\mathrm{y}$ a désormais un décalage entre la volonté des intellectuels de faire passer certains messages, et la fiabilité que leur attribue l'opinion publique. De nombreux Français cherchent alors à se rendre sur place. Certains y ont des attaches familiales et participent aux déplacements qui ont repris entre Français d'origine polonaise et Pologne après l'ouverture de $1956^{55}$. Mais le phénomène majeur en 1980 est le déplacement de Français sans origine polonaise, qui souhaitent découvrir ce pays si «familier» et où s'exprime un nouveau mouvement ouvrier. Jusque-là, ce type de voyage concernant des Français sans attache personnelle avec la Pologne était surtout le fait des intellectuels dans le cadre des échanges officiels. Mais là, il s'agit de départs «non maîtrisés », sinon par l'octroi ou le refus d'un visa. Les archives de certains syndicats comme la CFDT (Confédération française démocratique du travail) rassemblent de

${ }^{53}$ K. P o mi a n, interview, Faire, n 50, décembre 1979, p. 30.

54 Bibliothèque polonaise de Paris, Archives Lucienne Rey, Lettre du 14 février 1979.

55 M.-Cl. L o is o n, Les relations entre Solidarnosc et la CFDT, août 80-décembre 81, sous la direction d'A. Snejdarek, p. 69. 
nombreux témoignages de ces individus qui partent. Et très vite la CFDT s'interroge sur ces «voyages» touristiques. S'ils relèvent en partie d'une volonté de découvrir un pays exotique, ils témoignent aussi d'un engagement très militant. Il s'agit d'apporter enfin une aide concrète, matérielle aux Polonais. Bien souvent, la direction de la CFDT n'est contactée que pour participer aux frais ou pour obtenir des noms, des contacts en Pologne. C'est surtout début janvier 1981 que la direction de la CFDT constate le phénomène, d'une ampleur telle qu'elle suggère de proposer elle-même par les Comités d'entreprise des «voyages militant CFDT» avec des financements ${ }^{56}$. Un programme "d'échanges et de voyages entre Solidarność et la CFDT» se met en place, exprimant par là une volonté de canaliser les flux de militants.

En effet, les événements de juillet-août 1980 et la naissance du syndicat national Solidarność donnent une impulsion considérable à l'aide française. La lutte polonaise, incarnée par son chef emblématique, Lech Wałęsa, gagne le respect d'une grande partie des Français. Par son caractère simple, sa piété profonde et ses discours qui en appellent non pas à la révolte, comme l'histoire polonaise en a trop connu, mais à un dialogue ferme avec le pouvoir, il conquiert l'opinion publique internationale. Par le relais des médias, et lors de son déplacement en France du 14 au 21 octobre 1981, Lech Wałesa gagne une popularité essentielle pour obtenir le soutien de l'opinion publique française. En effet, le pouvoir polonais tient à garder ses liens, ne serait-ce qu'économiques avec la France. Or la période 1980-1981 est une période qui précède les élections présidentielles de mai 1981. Les dirigeants politiques sont très attentifs à l'évolution de l'opinion publique et au vote potentiel des électeurs. Particulièrement la gauche, qui a des chances de parvenir au pouvoir. Cet aspect modère la répression éventuelle à l'égard des opposants, et soutient la constitution du syndicat polonais. D'autant que des personnalités médiatiques s'engagent pour lui donner encore plus d'écho en France, comme Yves Montand qui reçoit la délégation polonaise le 20 octobre 1981. S'ensuivent d'autres manifestations très médiatisées, qui alimente l'implication massive des Français pour les Polonais, et en même temps en sont le reflet. De nombreux Français en effet constituent des colis qu'ils font passer en Pologne soit par des associations, soit tout simplement par des Polonais qui vont voir leurs familles. Les collectes de dons sont régulières, ne serait-ce que celle, très médiatisée, qui a lieu la journée du 12 décembre 1981 animée par Pierre Bellemare et Jacques Chirac pour «Le Noël des Polonais $»^{57}$. La proclamation de l'état de siège le lendemain, qui est annoncée par tous les médias français dès le dimanche matin, n'est pas à l'origine de la solidarité de nombreux français. Elle ne fait que renforcer leur décision d'aider par tous les moyens les Polonais. Cet énorme élan de sympathie, mobilisé par une émotion collective dont les ressorts seraient encore à établir ${ }^{58}$, a été rappelé dans des ouvrages polonais rassemblant de nombreux témoignages d'époque, de nombreuses sources ${ }^{59}$. En revanche, c'est du côté des intellectuels de gauche français, plus distanciés dans leur grand majorité à l'égard de Solidarność depuis sa création à l'été 1980, que le revirement est brutal. Il se

\footnotetext{
${ }^{56}$ Archives CFDT. carton 8H1919, document sur le projet d'échanges et de voyages dans le cadre des relations CFDT/S, du 13 janvier 1981.

${ }^{57}$ Libération, 8 décembre 1981, p. 6.

${ }^{58}$ Certaines pistes pour comprendre comment c'est construite l'émotion française autour de l'état de siège sont proposées dans notre travail de thèse sur les relations entre intellectuels français et polonais.

${ }^{59}$ Particulièrement l'ouvrage de M. Frybes, Merci pour votre solidarité, Institut Adam Mickiewicz, 2005.
} 
manifeste dans un premier temps par la manifestation massive qui a lieu à Paris - mais aussi dans toute la France - le 14 décembre 1981 pour la Pologne. La participation à ce rassemblement populaire en faveur de Solidarność et de la Pologne est une démarche fondatrice pour nombre d'entre eux. Tous les témoignages concernant la Pologne évoquent ce jour de décembre comme un moment extraordinaire, une rencontre exceptionnelle. Un engagement s'exprime de la part de personnalités a priori «non engagées », qui se faisaient un principe de ne pas accoler leur nom à une prise de position dans l'espace public, par exemple Jacques Le Goff ${ }^{60}$. Cette manifestation donne du côté français un sentiment de cohésion, de rapprochement unique après les erreurs et les remises en cause des engagements militants des années 1960 et 1970: en manifestant pour la Pologne, on ne peut pas se tromper. Celle-ci regroupe de manière exceptionnelle des personnes de milieux très différents, et de toutes sensibilités politiques. Si Paris mobilise le plus grand nombre de personnes, avec une estimation de «plusieurs dizaines de milliers » de manifestants pour la CFDT, les grandes villes atteignent autour de 10000 participants: par exemple à Grenoble, Marseille, Bordeaux ou Rennes. Des villes moyennes ont entre 2 et 4000 participants, comme Limoges, Pau, Dijon, Brest, Tours, Orléans, Reims, Besançon, Montpellier, Perpignan, Nancy, Lille, Cherbourg, Caen, Angers, Nice ou Saint-Étienne. Enfin une mobilisation égale ou inférieure à un millier est indiquée dans une soixantaine de villes françaises. La somme totale qui ressort de ces archives approche les 156000 manifestants ${ }^{61}$. Les intellectuels français sont alors confrontés à une réaction qui dépasse le cadre de leurs propres relations avec les intellectuels polonais. Il y a un nouvel enjeu, à savoir la place à regagner entre le pouvoir et l'opinion publique. Dès lors, la plupart d'entre eux va s'engager pour la Pologne. Ce seront d'abord Pierre Bourdieu et Michel Foucault qui lancent conjointement une protestation des intellectuels contre la passivité du pouvoir français. Les enjeux en sont aussi politiques : la Pologne encore une fois, est mobilisée par les intellectuels français pour interroger la gauche française et les socialistes. Le cas polonais donne lieu à des tensions dans les milieux intellectuels, notamment entre ceux qui faisaient partie de «La Deuxième gauche» et ceux qui soutiennent François Mitterrand. Les archives du quai d'Orsay montrent que, quelques mois avant l'état de guerre, ce dernier est conciliant avec le pouvoir polonais. La visite du ministre des affaires étrangères Claude Cheysson à Stanisław Kania a montré la volonté des autorités françaises d'être proches $\mathrm{du}$ gouvernement polonais ${ }^{62}$. Cet aspect peut expliquer la phrase que prononce Claude Cheysson le dimanche 13 décembre, «bien entendu nous ne ferons rien», et qui embrase de nombreux français. Face à la mobilisation de l'opinion publique française d'ailleurs, le gouvernement change de position début 1982. Les archives diplomatiques montrent que la réactivité populaire conduit les socialistes français à s'informer davantage sur la situation polonaise, et à infléchir pour un temps leur soutien au gouvernement polonais.

L'étude du transfert de la Pologne en France montre la complexité des transferts culturels, qui dépendent de nombreux facteurs. En prenant pour objet d'étude l'opinion publique et les intellectuels, on observe que le rapport à l'étranger diffère en fonction du

${ }^{60}$ Entretien de Jacques Le Goff avec l'auteur du 28 mai 2008.

${ }^{61}$ Paris, Archives CFDT, carton 8H1920.

${ }^{62}$ Archives du Ministère des Affaires étrangères, (AMAE), série Europe, sous-série Pologne, tranche de 1981 à 1985 , carton 5416 . 
bagage d'interprétation et de compréhension que chacun possède. Néanmoins, cela permet d'approfondir l'étude des intellectuels en France, de plus en plus contestés au début des années 1980 et dont les politiques - mais cela a aussi un rapport avec la Pologne en France - annoncent la «fin» ou «la mort». Cette étude a cherché à montrer aussi comment l'engagement pour la Pologne constitue une étape importante de la mobilisation collective avant l'ère du numérique qui en accélère le processus et l'individualisation.

\section{Bibliographie}

Archives:

Archives de la Confédération française démocratique du travail (CFDT, Carton 8H1919.

Fonds André Mazon, (Papiery André Mazona), Institut d'études slaves, Paris.

Archives du Ministère des Affaires étrangères, (AMAE), série Europe, sous-série Pologne, sygn. 5416.

Littérature:

Andréani J., Le piège, Helsinki et le monde communiste, Odile Jacob, Paris 2005.

Beauvois D., «L'accueil des Polonais en France après l'insurrection de 1830-1831 », [in:] Polonia, desPolonais en France de 1830 à nos jours, sous la direction Janine Ponty, Montag 2010.

Bernard M., Łaptos J., Les Relations entre la France et la Pologne au XX è s., Eventus, Kraków 2002.

Boudic G., Esprit 1944-1982, Les métamorphoses d'une revue, éditions de l'IMEC, Paris 2005.

Dubois D., « La Société historique et littéraire polonaise et la Biblioteka Polska de Paris », [in:] Polonia..., p. 30.

Frybes M., «L'Octobre polonais et ses conséquences pour les relations franco-polonaises », [in:] 1956, une date européenne, sous la direction de G. Mink, M. Lazar et M. J. Sielski, Éditions Noir sur Blanc, 2010.

Grémion P., « Voix d'exil en marge du progressisme parisien », [in:] Intellectuels de l'Est exilés en France, sous la dir. de W. Falkowski et A. Marès, Institut d'études slaves, Paris 2011.

Kuk L., " Les échanges culturels et scientifiques franco-polonais à l'épreuve de la guerre froide », Une Europe malgré tout, 1945-1990. Contacts et réseaux culturels, intellectuels et scientifiques entre Européens dans la guerre froide, sous la direction A. Fleury et L. Jilek, édition Peter Lang, Bruxelles 2009.

Lecomte B., La vérité l'emportera toujours sur le mensonge, J. C. Lattès, Paris 1991.

Łaptos J., « Le pacifisme apprivoisé : le congrès des intellectuels pour la défense de la paix en 1948 », [in:] Le pacifisme en Europe des années 20 aux années 1950, sous la direction Maurice Vaïsse, Bruylant, Paris 1993.

Marès A., " Aperçu des communautés centre-européennes à Paris dans l'entre-deux-guerres », [in:] Paris, " capitale culturelle » de l'Europe centrale?, p. 20.

Markiewicz Z., « Jean Potocki, auteur du Manuscrit trouvé à Saragosse, et ses liens avec les intellectuels français des Lumières ", [in:] Approches des Lumières: mélanges off erts à Jean Fabre, éd. Klincksieck, Paris 1974.

Ory P., Sirinelli J.-F., Les intellectuels en France. De l'affaire Dreyfus à nos jours, éditions Perrin, Paris 2004.

Pasztor M., Jarosz D., Conflits brulants de la guerre froide: les relations franco-polonaises de 1945 à 1954, Lavauzelle, Paris 2005.

Ponty J., Les Polonais en France, Editions du Rocher, 2008.

Ponty J., Polonais méconnus, Histoire des travailleurs immigrés en France dans l'entre-deux-guerres, Publications de la Sorbonne, Paris 2005.

Tollet D., «Introduction », La Pologne et l'Europe occidentale du Moyen-Âge à nos jours, Instytut Historii UAM, Poznań-Paris 2004.

de Villefosse L., « Réponse à Jean-Paul II », Contrepoints nr 29, 1979. 
Lidwine Warchol

\title{
Poland in France: from French public opinion to intellectuals
}

\begin{abstract}
Summary
The article deals with the relationship between two social actors, that is to say the French intellectuals and the French opinion, to Poland. This study is part of a work of crossed history, starting with the French context to show what circulates about Poland in France, and how. From there, the idea is also to see how the reception of Poland can modify or alter the interventions of one another. The study goes back to the origins of the representations on Poland which emerged either through old contacts established at the time of the Ancient Regime, or following the arrival of Polish immigrants in France, who have a very different understanding depending on their social background. If, for a long time, the relationship to these immigrants has forged the image of Poland in France, a far less positive image than some stereotypes suggest - these representations have been slowly modified thanks to the possibilities to travel and the opening of the country in the late $1950 \mathrm{~s}$. The 1970s and the issue of socialism coming to power in France allowed opposing Polish intellectuals to became a reference in France, on the public opinion's side, a whole Poland benefited a positive image, enhanced by the election of the pope. Finally, the Solidarność moment and the state of war showed how French intellectuals and French public opinion brought a different insight on this events and how the latter won over it thanks to the role of the collective emotion that then expressed.
\end{abstract}

Key words: Poland, France, intellectuals, political emigres, mutual relations. 